\title{
Variation in the Etch Rate of LIBWE Fabricating Deep Microtrenches
}

\author{
Tadatake SATO ${ }^{* 1}$, Yoshizo KAWAGUCHI ${ }^{* 1}$, Ryozo KUROSAKI ${ }^{* 1}$, Aiko NARAZAKI $^{* 1}$, \\ Wataru WATANABE ${ }^{* 1}$ and Hiroyuki NIINO*1 \\ ${ }^{* 1}$ Electronics and Photonics Research Institute, National Institute of Advanced Industrial Science \\ and Technology (AIST), Tsukuba Central 5, 1-1-1 Higashi, Tsukuba, Ibaraki 305-8565, Japan \\ E-mail: sato-tadatake@aist.go.jp
}

\begin{abstract}
Variation in the etch rates for fabricating single deep microtrenches by laser-induced backside wet etching (LIBWE) employing a $\mathrm{KrF}$ excimer laser and acetone solution of pyrene is investigated. Microtrenches with widths of $5,8,10,15$, and $20 \mu \mathrm{m}$, a length of $1 \mathrm{~mm}$, and depths up to $90 \mu \mathrm{m}$ were fabricated with a fluence of $1.3 \mathrm{~J} / \mathrm{cm}^{2}$, a pulse repetition of $80 \mathrm{~Hz}$, and various pulse numbers. Special care was directed to maintaining in-focus conditions for etching. In the fabrication of 5- $\mu \mathrm{m}-$ wide microtrenches, etch rates of $5.3 \mathrm{~nm} /$ pulse evaluated for trenches with depths of less than $4 \mu \mathrm{m}$ increased by a factor of 2.55 to $13.5 \mathrm{~nm} /$ pulse when the trenches became deeper than $16 \mu \mathrm{m}$. Such variation in the etch rate reduced with width and became negligibly small for 20 - $\mu$ m-wide microtrenches. Based on a similar dependence on width, the variation in etch rate with depth can be related to the size effect observed in LIBWE. As a factor causing the size effect as well as the variation in etch rate with depth, the interaction between the laser-heated surface and the solution in an early time region of the laser-induced phenomena is proposed. DOI:10.2961/jlmn.2012.01.0016
\end{abstract}

Keywords: Laser-micromachining, LIBWE, deep trench, high aspect ratio, etch rate variation

\section{Introduction}

Laser micromachining of transparent materials is widely studied as an important technique for fabricating micro electro-optical-mechanical system (MEOMS) components, microfluidic chips, and so on. Recently, indirect processing methods have proved to be an effective means of precise and low-damage micromachining of hard and brittle transparent materials; in these methods, the backside irradiation layout is generally employed. Laserinduced backside wet etching (LIBWE) uses a liquid as laser absorbing media [1-21]: generally an organic dye solution but a liquid metal is occasionally used instead $[10,17,19]$. Micromachining using inorganic solutions has been reported by Schafeev and co-workers [22-24]; in this method, a metal layer deposit on the irradiated area contributes to the etching. Recently, a similar approach was reported by Huang et al. [25]. Sugioka and co-workers $[26,27]$ developed the laser-induced plasma-assisted UV ablation (LIPPA) method. Laser induced backside dry etching (LIBDE) method [28,29] and laser etching at surface adsorbed layer (LESAL) [30] have also been studied. In these methods, a thin metal layer and an adsorbed layer from toluene vapor are employed as the laser-absorbing media, respectively.

Indirect processing like LIBWE utilizes transient phenomena induced at the liquid-solid interface. Therefore, it is basically a surface machining technique. Owing to the utilization of a liquid as the laser-absorbing media in LIBWE, fabrication of deep microstructures with high aspect ratios becomes possible: this is a prominent characteristic of this technique [2,31]. At present, a deep trench with an aspect ratio as high as 100 has been successfully fabricated [32]. Because of the limited depthof-focus (DOF) of the projection system, the position of the sample must be continuously optimized to keep the location of the glass-liquid interface within the DOF. For this optimization, information on the etch rate is indispensable. In the fabrication of such deep microstructures, we have noticed unexpected variations in the etch rate: an increase in the etch rate was observed in the fabrication of a deep microtrench with a width of 10 $\mu \mathrm{m}$ under constant conditions for irradiation [33]. The observed variation in the etch rate may be due to different spatial conditions surrounding the irradiated area.

Despite the intensive studies so far, the LIBWE mechanism is still not fully understood. In the initial stage of LIBWE, laser light passing through the glass is completely absorbed by the very thin liquid layer at the interface to form a transient photo-activated region. This region acts on the surface layer of the glass, resulting in etching as well as the generation of shockwaves and cavitation bubbles $[6,15]$. Meanwhile, the contribution of a thin amorphous carbon layer generated by thermal degradation of the heated solution to the etching process has been pointed out $[5,8,9,14]$, and the contribution of the high absorbing modified fused silica has also recently been presented [11,12].

As described above, possible fabrication of microstructures with high aspect ratios is a prominent characteristic of LIBWE, and information on the precise etch rate is important for the fabrication. Moreover, a detailed study of the observed variation in the etch rate can serve to provide new phenomenological findings that contribute to the understanding of the LIBWE mechanism. In this work, we have investigated, in detail, the variation in the etch rate for fabricating deep microtrenches with various widths. 


\section{Experimental}

Microstructures were fabricated on synthetic silica glass plates (Tosoh SGM, ES grade, size: $2 \times 2 \mathrm{~cm}$, thickness: $2 \mathrm{~mm}$ ) by LIBWE employing an acetone solution saturated with pyrene and $\mathrm{a} \mathrm{KrF}$ excimer laser (Lambda Physik, Compex110, $\lambda=248 \mathrm{~nm}, \mathrm{FWHM} \approx$ $30 \mathrm{~ns}$ ). The repetition rate and fluence were set to $80 \mathrm{~Hz}$ and $1.3 \mathrm{~J} \cdot \mathrm{cm}^{2} \cdot$ pulse $^{-1}$, respectively. Microstructures were generated by using a mask projection system with a nominal 1/8 demagnification including a variable attenuator and beam homogenizer (MicroLas, ValioLas). The nominal optical resolution of this system is specified as followed: $3 \mu \mathrm{m}$ lines and spaces (diffraction limit). To set the laser fluence, the laser light power passing through a circular diaphragm with a diameter of $7 \mathrm{~mm}$, which is set at the position of the photomasks, was measured at a position just after the projection lens by means of a power meter. After the laser fluence was adjusted by means of the variable attenuator, the circular diaphragm was replaced by the photomasks. By using chromium-on-quartz-masks with a rectangular opening, a rectangular area at the liquid-solid interface was irradiated for etching. For instance, a mask with a $80 \times 8000 \mu \mathrm{m}$ opening was used for fabricating 10 $\mu \mathrm{m}$-wide microtrenches with a length of $1 \mathrm{~mm}$. The location of the micromachining was controlled using a computer-controlled XYZ stage (Sigmatech Co., FS-1050), on which a sample composed of a glass plate and solution was mounted. The position of the sample was controlled with a resolution of $\pm 0.2 \mu \mathrm{m}$, and the shifting speed can be set with a resolution of $0.1 \mu \mathrm{m} \cdot \mathrm{s}^{-1}$.

To observe the cross-sectional images of the trenches, the glass plates on which trenches were fabricated were cut perpendicular to the long axis of the trenches. To cut at the center of trenches (1 mm length), guiding trenches $(10 \mu \mathrm{m}$ wide and ca. $350 \mu \mathrm{m}$ deep) were also prepared by LIBWE. Cross-sectional images were obtained with a digital optical microscope (Keyence, VH-5500 with VHZ-100R) and a scanning electron microscope (SEM, Keyence, VK-7800).

\section{Results}

For fabricating deep microtrenches, the glass-liquid interface to be etched must be continuously located within the DOF of the projection system [2,31]. Since the position of the projection lens is fixed in our system, the sample position must be adjusted. First, the variation in the depth of microtrenches fabricated with constant pulse numbers at various positions along the optical axis was investigated. Figure 1 shows the results for microtrenches with a width of $5 \mu \mathrm{m}$. All microtrenches were fabricated on a pristine surface with 153 pulses. The depths of the microtrenches fabricated at positions between -16 and $+16 \mu \mathrm{m}$ were nearly constant $(0.707 \pm 0.016 \mu \mathrm{m})$. Microtrenches with other widths $(8,10,15$, and $20 \mu \mathrm{m})$ also showed nearly constant depths in this region (results not shown here). Thus, it was confirmed that the etch rate remains constant when the liquid-glass interface is located within a ca. 30 $\mu \mathrm{m}$ range of the DOF.

As the etching proceeds, the position of the glass-liquid interface moves into the glass substrate. To fabricate deep microtrenches, the sample position must be dynamically adjusted to maintain the position of the glass-liquid interface within the DOF. Additionally, since the image is formed on the backside surface of the glass, the position for in-focus etching is affected by the thickness of the remaining glass layer. Figure 2 shows the positions for infocus etching of silica glasses with different thickness.

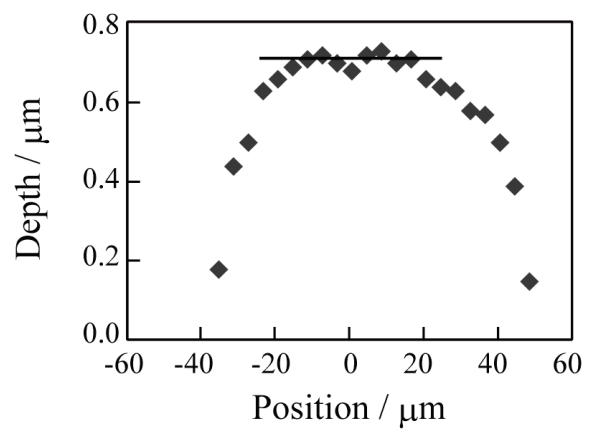

Fig. 1 Depths of 5- $\mu$ m-wide microtrenches fabricated with 153 pulses at various positions along the optical axis. The position denoted by zero is the infocus LIBWE position for silica glass with a thickness of $2 \mathrm{~mm}$. A positive position denotes a position closer to the projection lens.

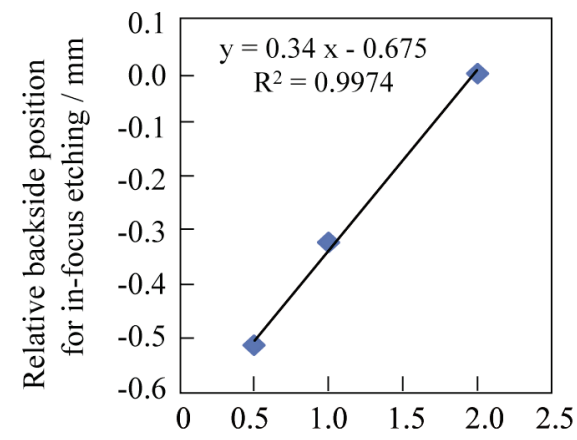

Thickness of glass plate / $\mathrm{mm}$

Fig. 2 Relative backside positions for in-focus etching of silica glasses with different thickness. Negative values indicate a decrease in the working distance from the projection lens. The fitting result is also shown.

When an image is formed through a silica glass plate with a refractive index $n$ and thickness $t$, the working distance from the projection lens is elongated by $\delta$.

$$
\delta=(1-1 / n) t
$$

For silica glass with $n=1.50841$ at $\lambda=248 \mathrm{~nm} \mathrm{[34],}$ the value, $(1-1 / n)=0.33705$, shows good agreement with the inclination of 0.34 shown in Fig. 2. When a structure with a depth of $d$ is formed, the thickness of the glass reduces to $(t-d)$. Since the backside surface of the glass is located at a distance $d$ from the bottom of the structure, the required shift from the original position is $+d / n$ to maintain in-focus etching.

$$
\delta^{\prime}=(1-1 / n)(t-d)=\delta-d+d / n
$$

The conditions for the stage shift were determined as follows: (1) microtrenches with various depths were fabricated without stage shift, (2) the depths of the 
fabricated microtrenches were evaluated, and (3) the conditions for the stage shift were set on the basis of the obtained results. The microtrenches were then fabricated under the stage shifted conditions. If the deviation between the bottom of the trench and the image plane is larger than $10 \mu \mathrm{m}$, the conditions for the stage shift were revised, and microtrenches were fabricated under the revised conditions. Figure 3 shows the results obtained for $10-\mu \mathrm{m}$ wide microtrenches. The solid line denotes the position of the image plane estimated on the basis of the stage motion and refractive index of silica glass. As the figure shows, the deviation between the position of the bottom of the trench and that of the image plane remained less than $10 \mu \mathrm{m}$, indicating that in-focus etching was performed continuously.

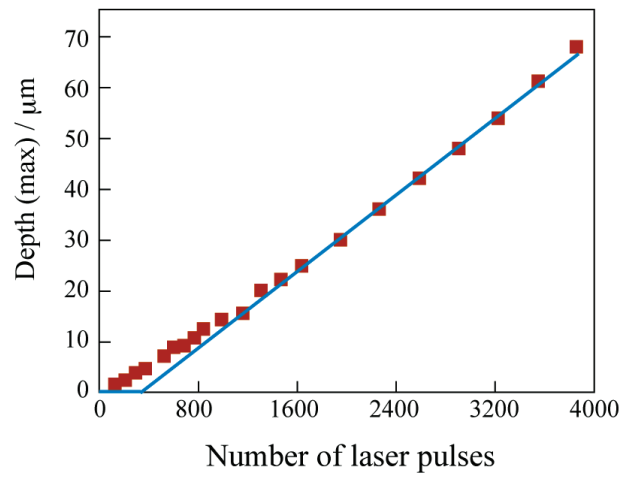

Fig. 3 Etch depths of 10- $\mu$ m wide trenches fabricated with various irradiation times. The solid line shows the position of the image plane.

Despite the continuous etching under in-focus conditions, the depths of the trenches clearly show two inclinations indicating different etch rates. An increased etch rate has been observed for trenches deeper than ca. $25 \mu \mathrm{m}$. Two etch rates of 14.8 and $19.0 \mathrm{~nm} /$ pulse were estimated for shallow (denoted by S) and deep (denoted by D) microtrenches, respectively, as shown in Figure 4. The limits of the regions were determined arbitrarily from the graphs. The etch rate for the deeper trenches was evaluated to be 1.25 times larger than that for shallow trenches with depths of less than $10 \mu \mathrm{m}$.

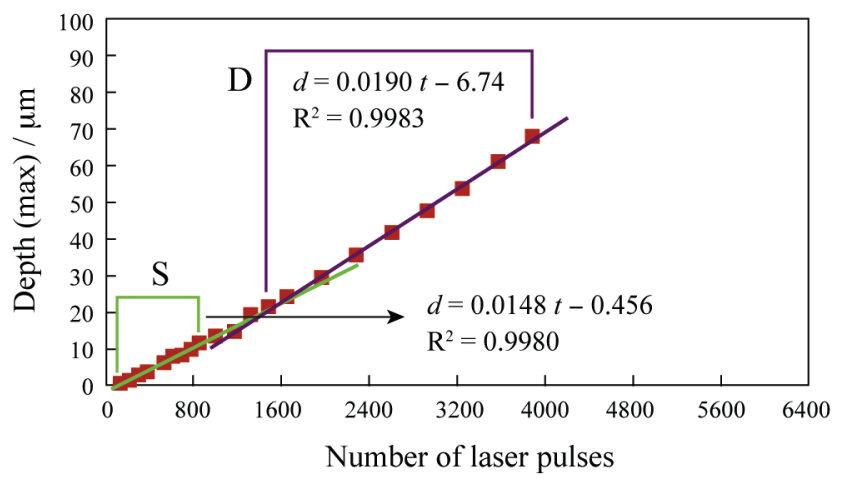

Fig. 4 Etch rates estimated for 10 - $\mu \mathrm{m}$ wide trenches in the two regions of irradiation with (S) 100-840, and (D) 1460-3900 pulses, respectively. Fitting results are also shown.
These etch rates were confirmed to be reproducible within experimental error in at least three sets of experiments. The $y$-intercept of the fitted line for the shallow trenches was not equal to zero, indicating the existence of incubation in LIBWE [9].

The etch depths for fabricating microtrenches with widths of 5, 8, 15, and $20 \mu \mathrm{m}$ are shown in Figure 5.
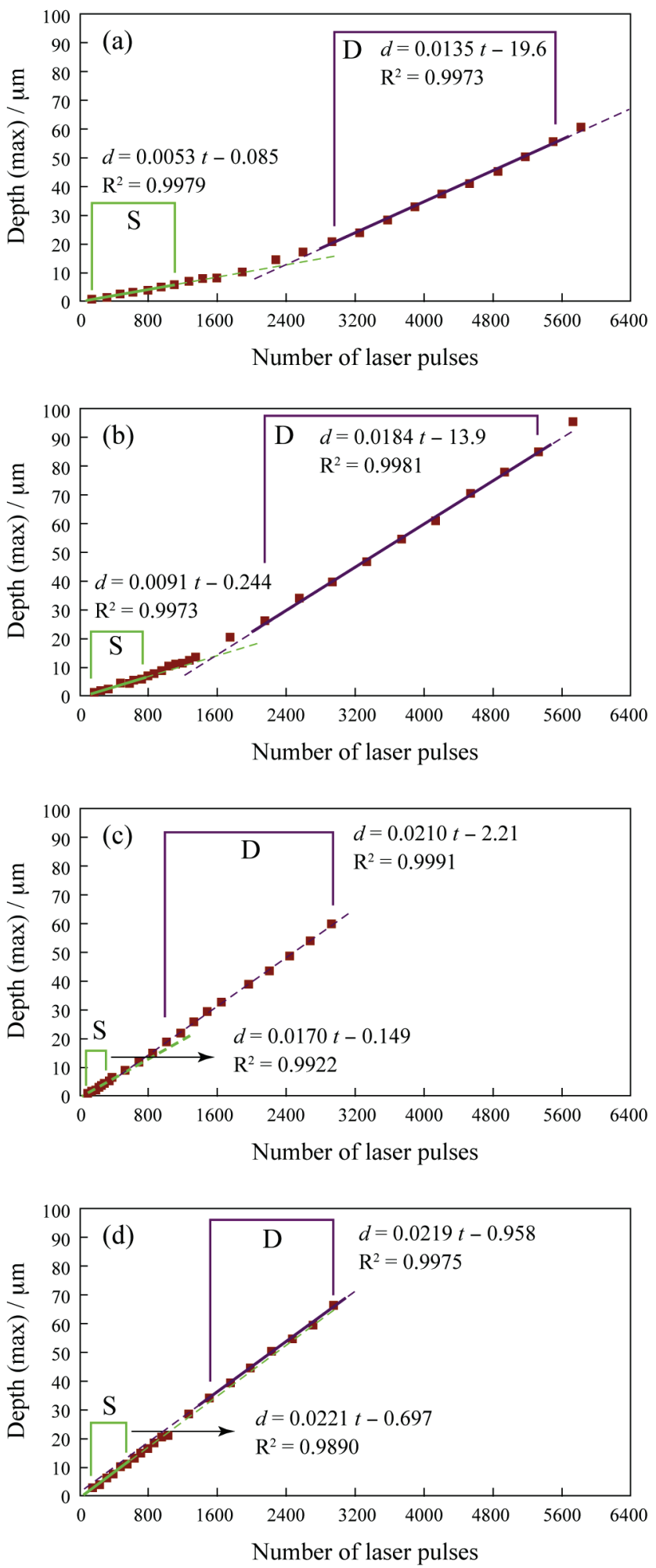

Fig. 5 Depths of (a) $5-\mu \mathrm{m}$, (b) $8-\mu \mathrm{m}$, (c) $15-\mu \mathrm{m}$, and (d) $20-\mu \mathrm{m}$ wide microtrenches and the evaluation of the two etch rates within the shallow (S) and deep (D) ranges. Fitting results are also shown. 
In all cases, the deviation between the position of the bottom of the trench and that of the image plane was maintained to be less than $10 \mu \mathrm{m}$. Reproducibility was similarly confirmed for these widths. Two inclinations for the different etch rates were clearly observed in the depths of the 5- and 8- $\mu \mathrm{m}$ wide microtrenches (Figure 5(a) and (b)). The etch rates for shallow trenches (Es), those for deep trenches (Ed), and the increasing ratios $(\mathrm{Ed} / \mathrm{Es})$ for the microtrenches with various widths are summarized in Table 1 together with the depths $\left(\mathrm{IS}_{\mathrm{D}}\right.$, the corresponding aspect ratios shown in parentheses) and pulse numbers $\left(\mathrm{IS}_{\mathrm{PN}}\right)$ for the intersections of the two fitted lines. The maximum variation in the etch rate was observed for $5-\mu \mathrm{m}$ wide microtrenches: the etch rate for deep trenches was 2.55 times larger than that for shallow trenches. The variation in the etch rate reduced with width and becomes almost negligible at a width of $20 \mu \mathrm{m}$.

Table 1 Etch rates and related results obtained for microtrenches with various widths

\begin{tabular}{|c|c|c|c|c|c|}
\hline $\mathrm{Width} / \mu \mathrm{m}$ & 5 & 8 & 10 & 15 & 20 \\
\hline $\begin{array}{c}\text { Es } \\
/ \mathrm{nm} \mathrm{pulse}^{-1}\end{array}$ & 5.3 & 9.1 & 14.8 & 17.0 & 22.1 \\
\hline $\begin{array}{c}\mathrm{Ed} \\
/ \mathrm{nm} \mathrm{pulse}^{-1}\end{array}$ & 13.5 & 18.4 & 19.0 & 21.0 & 21.9 \\
\hline $\mathrm{Ed} / \mathrm{Es}$ & 2.55 & 2.02 & 1.25 & 1.24 & 0.99 \\
\hline $\mathrm{IS}_{\mathrm{D}} / \mu \mathrm{m}$ & $13(2.6)$ & $13(1.6)$ & $22(2.2)$ & $9(0.6)$ & - \\
\hline $\mathrm{IS}_{\mathrm{PN}} /$ pulse & 2380 & 1468 & 1496 & 515 & - \\
\hline
\end{tabular}

The $\mathrm{IS}_{\mathrm{D}}$ values do not show a clear dependence on the width. For the 5- and 8- $\mu \mathrm{m}$ wide microtrenches, a difference between the two inclinations is clearly observed. The same $\mathrm{IS}_{\mathrm{D}}$ value of $13 \mu \mathrm{m}$ was found for these cases, despite the $I_{\mathrm{D}}$ corresponding to different aspect ratios of 2.6 and 1.6. Meanwhile, for the $10-$ and $15-\mu \mathrm{m}$ wide microtrenches, the difference between the two etch rates observed for the shallow and deep trenches is small. Because of this small difference and the arbitrarily determined limits of the regions, the results on the intersections of the two fitted lines might include an artifactual error. These results indicate that factors causing the variation in the etch rate do not originate from the phenomena affected by the aspect ratio of the fabricated structure. Moreover, the $\mathrm{IS}_{\mathrm{D}}$ values, indicating the region where the phenomena causing the variation in etch rate occurs, are much larger than the penetration depth of the laser light in the solution, which is about $1 \mu \mathrm{m}$.

\section{Discussion}

Increased etch rates observed for deep microtrenches are unexpected results: when microtrenches become deeper, the removal of the etched silica from the trenches becomes difficult because of the longer distance over which the silica is transported. If resolidification of the etched silica to sidewalls or the bottom of the trenches occurs, it decreases the etching efficiency. However, increased etch rates were observed in deeper microtrenches. In addition, no notable change in width was observed for deep microtrenches [32]. Efficient mass transportation is demonstrated by the reproducible generation of deep microtrenches with homogeneous widths. Thus, it is concluded that resolidification is a minor process in the fabrication of deep microtrenches. Fused silica removed from the bottom of deep microtrenches must be trapped in solution and expelled from the trench by the motion of a cavitation bubble as discussed in the previous report [31]. Similar action of the liquid is often utilized in waterassisted ablation with femtosecond laser pulses [35,36].

The dependence of the etch rate on the width of the microtrenches is depicted in Figure 6. In addition to the results for trenches with widths less than $20 \mu \mathrm{m}$, the etch rates for fabricating shallow trenches with widths of 25,30 , and $50 \mu \mathrm{m}$ were evaluated to be $23.8,24.4$, and $24.4 \mathrm{~nm} /$ pulse, respectively. The observed dependence on the width is similar to those for the reported size effect in LIBWE $[9,37]$. As shown in Figure 6 , both etch rates obtained for shallow and deep microtrenches exhibit a similar dependence on width. These results indicate that the variation in etch rate with depth has the same origin as the size effect in LIBWE.

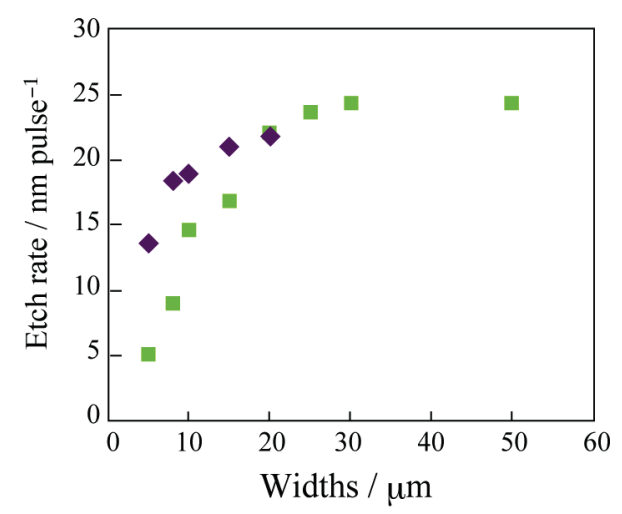

Fig. 6 Etch rates evaluated for deep (diamonds) and shallow (squares) microtrenches with various widths.

In early work, Wang et al. [37] ascribed the thermal energy loss from the liquid layer absorbing the laser energy to the factors that caused the size effect. In this work, a decrease in the etch rate was observed for trenches with widths less than $30 \mu \mathrm{m}$. Since this size is much larger than the thermal diffusion lengths of fused silica and solution, which are less than $1 \mu \mathrm{m}$, thermal energy loss is not an adequate factor to cause the size effect. Böhme and Zimmer [9] proposed the action of the cavitation bubble, which is transiently produced in the solution and has a size larger than the thermal diffusion lengths, as the factor causing the size effect. This proposal is based on two assumptions: (1) the thickness of the amorphous carbon film generated on the surface is proportional to the lifetime of the cavitation bubble, and (2) the etch rate of LIBWE using this film as a major laser absorber is proportional to the thickness of the film. Recently, they have reported that the modified fused silica acting as an additional laser absorber is responsible for the erosion process in LIBWE [12]. Considering the surrounding spatial conditions for the surface layers, the difference between LIBWE at the surface and at the bottom of the trench is small: one surface of the layer is in contact with solution and the other is in contact with the glass substrate. Such surface layers are formed by the interactions between the laser-heated surface 
and the organic molecules in solution. An amorphous carbon film on silica glass was reported to be generated by pyrolytic laser-induced chemical vapor deposition [38]. Meanwhile, a silicone suboxide assigned as a main component of the surface-modified layer was reported to be formed at temperatures higher than $1200{ }^{\circ} \mathrm{C}$ by the reaction of fused silica with hydrogen [39]. Given that a high cooling rate of more than $10^{9} \mathrm{~K} \cdot \mathrm{s}^{-1}$ has been numerically estimated for the laser-heated surface [12], it would be difficult to maintain the temperature for surface modification and deposition of amorphous carbon during the lifetime of the bubble, which is on the order of hundreds of microseconds. Meanwhile, the dynamics of the cavitation bubble can be described by the Rayleigh-Plesset equation. Recently, this model was modified to describe the cavitation bubble generated in liquid-phase laser ablation [40]. In this case, a semispherical bubble is generated at the liquid-solid interface as in the case of LIBWE [3]. This model indicates that the pressure inside the bubble decreased drastically as the bubble expands. Considering the dynamics of the pressure and temperature, the initial assumptions on the relationship between the lifetime of the cavitation bubble and the thickness of the amorphous carbon film should be revised. The surface layers contributing to LIBWE should be generated in an early time region of the laser-induced phenomena: in this time region, the temperature of the laser-heated surface is high enough for thermal reactions, and the heated solution is in a compressed condition before expansion. If bigger bubbles remain for longer in the compressed condition, they may interact for a longer time with the sample surface, and this can cause the formation of thicker surface layers and resultantly cause an increase in the etch rate of LIBWE. Moreover, it was reported that the surrounding confinement conditions retarded the dynamics of cavitation bubbles [41]. The bubble dynamics generated at the bottom of a deep trench would show similar retarded dynamics and results over a longer interaction time. This elongated interaction time can be ascribed to the enhanced thickness of the surface layers causing an enhanced etch rate for deep trenches.

Meanwhile, the impulse by the shock wave generated by laser ablation at the liquid-solid interface has been studied in terms of laser shot peening. Under confined geometry, which means the target covered by water, a shock wave with a larger pressure and longer duration can be generated; the pressure is more than an order of magnitude greater than ablation without confinement by water, and the duration of shock waves is 2-3 times longer in comparison with unconfined cases [42,43]. Since the impulse has a longer duration than the laser pulse, it interacts with the molten silica surface. Even if the material erosion is dominated by evaporation of the heated modified layer, the impact can result in the reshaping of the molten glass to form a round bottom and the removal of a part of the molten silica. Given that the induced maximum pressure is proportional to the square root of the laser power and the confinement effect by the glass is larger than that by water, the efficiency of the removal of the molten material can change with the size of the irradiated area as well as by the conditions surrounding the irradiated area.
As discussed above, the increased thickness of the surface layers and increased impulse can cause the size effect in LIBWE as well as the variation in the etch rate with depth. To clarify which factors actually affect the etch rate, the following verification would be necessary: (1) an investigation of the surface layer thickness generated at the surface and at the bottom of deep microtrenches with various widths, (2) an investigation of the pressure of the impact generated by the laser pulse incidence at the surface and at the bottom of deep microtrenches.

\section{Conclusions}

Possible fabrication of microstructures with high aspect ratios is a prominent characteristic of LIBWE. Information of the precise etch rate is important because the sample position must be dynamically adjusted to maintain the liquid-glass interface within the limited DOF of the projection system. Any unexpected variation in the etch rate observed during the fabrication of a deep microtrench must be taken into account by adjusting the sample position.

In this work, the maximum variation was observed for 5 - $\mu \mathrm{m}$-wide microtrenches. Etch rates of $5.3 \mathrm{~nm} /$ pulse were evaluated for trenches with depths less than $4 \mu \mathrm{m}$, and this increased by a factor of 2.55 to $13.5 \mathrm{~nm} /$ pulse when the trenches became deeper than $16 \mu \mathrm{m}$. Such variation in the etch rate reduced with width and became negligibly small for $20-\mu \mathrm{m}$-wide microtrenches. Both etch rates evaluated for shallow and deep trenches increased with width, indicating that the observed variation in the etch rate with depth has the same origin as the size effect in LIBWE and that the variation is due to the different spatial conditions surrounding the irradiated area. It is not adequate for the origin of the etch rate variation to be assigned to thermal effects because of the different scale of the thermal diffusion lengths of the materials. The variation in the etch rate depends on the irradiated area as well as the geometrical confinement and can be assigned to the phenomena induced in the heated liquid layer. As a factor that causes the size effect as well as the variation in etch rate with depth, we proposed the interaction between the laser-heated surface and the solution in the early time region of the laser-induced phenomena. The variation of the etch rate can be explained as a result of the enhanced surface layer thickness or the enhanced efficiency of the molten silica removal. To clarify which factor actually affects the etch rate, further verification would be necessary.

\section{References}

[1] J. Wang, H. Niino, and A. Yabe: Appl. Phys. A, 68, (1999) 111.

[2] Y. Kawaguchi, T. Sato, A. Narazaki, R. Kurosaki, and H. Niino: Jpn. J. Appl. Phys., 44, (2005) L176.

[3] H. Niino, Y. Yasui, X. Ding, A. Narazaki, T. Sato, Y. Kawaguchi, and A. Yabe: J. Photochem. Photobiol. A: Chem., 158, (2003) 179.

[4] X. Ding, Y. Yasui, Y. Kawaguchi, H. Niino, and A. Yabe: Appl. Phys. A, 75, (2002) 437.

[5] X. Ding, T. Sato, Y. Kawaguchi, and H. Niino: Jpn. J. Appl. Phys., 42, (2003) L176. 
[6] H. Niino, Y. Kawaguchi, T. Sato, A. Narazaki, T. Gumpenberger, and R. Kurosaki: Appl. Surf. Sci., 252, (2006) 4387.

[7] R. Böhme, A. Braun, and K. Zimmer: Appl. Surf. Sci., 196, (2002) 276.

[8] K. Zimmer, A. Braun, and R. Böhme: Appl. Surf. Sci., 208-209, (2003) 199.

[9] R. Böhme, and K. Zimmer: Appl. Surf. Sci., 247, (2005) 256.

[10] K. Zimmer, R. Böhme, D. Ruthe, and B. Raushenbach: Appl. Phys. A, 84, (2006) 455.

[11]K. Zimmer, R. Böhme, D. Ruthe, and B. Raushenbach: Appl. Surf. Sci., 253, (2007) 6588.

[12]K. Zimmer, M. Ehrhardt, and R. Böhme: J. Appl. Phys., 107, (2010) 034908.

[13] G. Kopitkovas, T. Lippert, C. David, A. Wokaun, and J. Gobrecht: Microelectron. Eng., 67-68, (2003) 438.

[14] G. Kopitkovas, V. Deckert, T. Lippert, F. Raimondi, C. W. Schneider, and A. Wokaun: Phys. Chem. Chem. Phys., 10, (2008) 3195.

[15]Cs. Vass, T. Smausz, and B. Hopp: J. Phys. D: Appl. Phys., 37, (2004) 2449.

[16]C. Vass, K. Osvay, T. Veso, B. Hopp, and Z. Bor: Appl. Phys. A, 93, (2008) 69.

[17]B. Hopp, T. Smausz, T. Csizmadia, C. Vass, T. Csako, and G. Szabo: J. Laser Micro/Nanoeng., 5, (2010) 80.

[18]J.-Y. Chen, M.-H. Yen, and T.-H. Young: J. Micromech. Microeng., 16, (2006) 2420.

[19] M.-H. Yen, C.-W. Huang, W.-C. Hsu, T.-H. Young, K. Zimmer, and J.-Y. Cheng: Appl. Surf. Sci., 257, (2010) 87

[20]K. Fujito, T. Hashimoto, K. Samonji, J. S. Speck, and S. Nakamura: J. Cryst. Growth, 272, (2004) 370.

[21]T. Lee, D. Jang, D. Ahn, and D. Kim: J. Appl. Phys., 107, (2010) 033112.

[22] S. I. Dolgaev, A. A. Lyalin, A. V. Simakin, and G. A. Shafeev: Quantum Electron., 26, (1996) 65.

[23] S. I. Dolgaev, A. A. Lyalin, A. V. Simakin, and G. A. Shafeev: Appl. Surf. Sci., 96-98, (1996) 491.

[24]A. V. Simakin, E. N. Lubnin, and G. A. Shafeev: Quantum Electron., 30, (2000) 263.
[25]Z. Q. Huang, M. H. Hong, T. B. M. Do, and Q. Y. Lin: Appl. Phys. A, 93, (2008) 159.

[26] J. Zhang, K. Sugioka, and K. Midorikawa: Appl. Phys. A, 67, (1998) 545.

[27] Y. Hanada, K. Sugioka, I. Miyamoto, and K. Midorikawa: Appl. Surf. Sci., 248, (2005) 276.

[28]B. Hopp, Cs. Vass, and T. Smausz: Appl. Surf. Sci., 253, (2007) 7922.

[29]R. Böhme, K. Zimmer, and B. Rauschenbach: Appl. Phys. A, 82, (2006) 325.

[30]R. Böhme, and K. Zimmer: Appl. Surf. Sci., 239, (2004) 109.

[31] Y. Kawaguchi, T. Sato, A. Narazaki, R. Kurosaki, and H. Niino: J. Photochem. Photobiol. A: Chem., 182, (2006) 319.

[32] T. Sato, R. Kurosaki, A. Narazaki, Y. Kawaguchi, and H. Niino: Proc. SPIE, 7584, (2010) 758408.

[33] T. Sato, R. Kurosaki, Y. Kawaguchi, A. Narazaki, and H. Niino: J. Laser Micro/Nanoeng., 5, (2010) 256.

[34]E. D. Palik, Ed., "Handbook of Optical Constants of Solid," (Academic Press, London, 1985).

[35] Y.Li, K. Itoh, W. Watanabe, K. Yamada, D. Kuroda, J. Nishii, and Y. Jiang: Opt. Lett., 26, (2001) 1912.

[36] Y. Li, S. Qu, and Z. Guo: J. Miromech. Microeng., 21, (2011) 075008.

[37]J. Wang, H. Niino, and A. Yabe: Proc. SPIE, 3933, (2000) 347.

[38] K. H. Kwok, and W. K. S. Chiu: Carbon, 41, (2003) 2307.

[39] S. T. Tso, and J. A. Pask: J. Am. Ceram. Chem., 65, (1982) 457.

[40] W. Soliman, T. Nakano, N. Takada, and K. Sasaki: Jpn. J. Appl. Phys., 49, (2010) 116202.

[41]P. A. Quinto-Su, K. Y. Lim, and C.-D. Ohl: Phys. Rev. E, 80, (2009) 047301.

[42] R. Fabbro, J. Fournier, P. Ballard, D. Devaux, and J. Virmont: J. Appl. Phys., 68, (1990) 775.

[43]D. Devaux, R. Fabbro, L. Tollier, and E. Bartnicki: J. Appl. Phys., 74, (1993) 2268.

(Received: June 6, 2011, Accepted: January 12, 2012) 